\title{
Il sistema dei Casali Fortezza. Il caso studio di Castellaccio di Monteroni
}

The Casali Fortezza system. The case study of Castellaccio di Monteroni

\author{
Daniele Calisi $^{\text {a, }}$, Maria Grazia Cianci ${ }^{\text {b }}$, Matteo Molinari ${ }^{\mathrm{c}}$ \\ Università degli Studi Roma Tre, Rome, Italy \\ adaniele.calisi@uniroma3.it; 'bariagrazia.cianci@uniroma3.it; c matteo.molinari@uniroma3.it
}

\begin{abstract}
The landscape of Lazio's Tyrrhenian coasts is strongly characterized by the presence of fortifications. Parallel to them, in the interland, the baronial expansion, between the tenth and eleventh centuries, paved the way to a large-scale fortification of the Roman countryside. Along the main routes were built lookout towers, farmhouses were consolidated with defense mechanism and the first castles were constructed. The research focuses on the study of Castellaccio di Monteroni in Ladispoli, built in the fifteenth century on Roman structures and on previous constructions dating back to the period of the Baronial expansion. The Castellaccio di Monteroni is one of the few remaining examples of casale fortificato (fortified manor). It is placed on the $35^{\text {th }} \mathrm{km}$ of the Via Aurelia and takes its name "Castellaccio" from the abandonment over the centuries and "Monteroni" due to the heaps of the nearby Etruscan necropolis. The main function carried out over the centuries was a resting place, a sure point of reference for couriers, travelers and pilgrims traveling along the Via Aurelia. When in the nineteenth the route of the Via Aurelia was moved to the present one the fortification fell into abandonment. The analysis of this architecture is of great interest, not only for the knowledge of the geometry of the fortifications of the Roman countryside, but also because it is one of the few remaining examples of casale fortificato, representing a rare medieval architectural heritage.
\end{abstract}

Keywords: Digital reconstruction, survey, casali, heritage.

\section{Introduzione}

La campagna romana è caratterizzata dalla presenza di più di 100 casali, un'eredità minima in confronto all'originario numero di 400 manufatti (Coste, 1969). Il deteriorarsi di queste architetture è notevolmente diverso rispetto ai secoli scorsi. Nell'ultimo secolo l'urbanizzazione e quindi il progressivo abbandono della campagna hanno portato a un forte disinteresse nei confronti di questi manufatti. In molti casi l'architettura originaria è andata persa sia per una distruzione volontaria del manufatto sia per il riammoderna- mento della struttura per adempire a funzioni diverse (Cosenza, 2017). È interessante constatare come il fenomeno dell'abbandono della campagna romana è, in effetti, contrario rispetto a quello avvenuto nella campagna rurale del centro Italia, dove si installò un altro sistema secolare di gestione: la mezzadria. Nell'usare il termine casale bisogna fare attentione nel non incorrere nell'errore di confonderlo con la tipologia architettonica della cascina. Mentre la cascina è una struttura a corte dedicata all'allevamento di ani- 
mali, il casale è un agglomerato di edifici isolati, nella campagna romana, dedito ai soli fini residenziali. Il casale per sua definizione è privo di mura o altri elementi architettonici volti a un fine difensivo, fanno eccezione i casali medievali presenti in Puglia.

Nel Lazio, e nello specifico nella campagna romana, l'origine dei casali risale al XII secolo. In seguito nel XIII diventa un' architettura comune per poi perdere sempre più di importanza nel XIV secolo. Il fenomeno di costruzione dei casali nel Lazio, sulle principali vie di comunicazioni, le consolari, viene definito incasalamento.

Gli studi di Carocci e Vendittelli hanno identificato come "Campagna romana" quella fascia attorno alla città estesa per circa 18-20 km (30 km nella zona costiera) in cui all'inizio del XII secolo erano presenti castelli fondati a partire dal IX secolo (Cardocci, Venditelli, 2004). Questa area fu invece interessata nei secoli seguenti da una costruzione di castelli e casali con caratteristiche differenti tra di loro e divisi in tre fasce concentriche. Una prima fascia, esterna a quella delle vigne e degli orti intorno alle Mura Aureliane, caratterizzata da 6-8 km esclusivamente composta da casali. Una seconda fascia, di 4-6 km, con casali e castelli più vicini a Roma. Una terza fascia, che arrivava fino ai confini della Campagna romana, con prevalente presenza di castra.

I principali attori di questa operazione sono state le élite ecclesiastiche, aristocratiche e mercantili romane. I casali della regione, a differenza dei tradizionali casali della penisola presentavano nella maggior parte dei casi una torre, posizionata o al centro della struttura o su uno dei lati del corpo di fabbrica. La torre aveva sia scopo difensivo sia di rappresentanza per la famiglia che vi dimorava (Bagnarini, 2013, pp. 99-118).

La funzione principale delle torri era quindi di tipo difensivo, ma all'interno vi si svolgevano anche funzioni utilitarie. Non sembra invece dimostrabile che esistesse una funzione volta alla comunicazione, una rete di posti di avvistamento in collegamento visivo tra di loro. Nei documenti a datazione medievale compare anche spesso il termine castellarium, diffuso sia per i casali che per i castelli, che andava a identificare gli edifici al centro del complesso. Altro elemento spesso presente erano le grotte naturali o pozzi artificiali nei dintorni o all'interno della struttura anch'esse utilizzate come magazzini o ricoveri.

La tipologia classica di casale presentava una sola torre, vi sono però testimonianze di architetture con due torri. Questa compresenza di elementi è dovuta probabilmente all'unione di più fabbricati o all'annessione di più famiglie sotto un'unica dimora. La torre risultava quindi l'elemento di maggior riconoscibilità dell'azienda agricola o della famiglia mercantile che vi dimorava, tanto che il termine casale viene utilizzato solo in un secondo momento per identificare queste architetture che prima erano definite case-torri (Rossi, 1969).

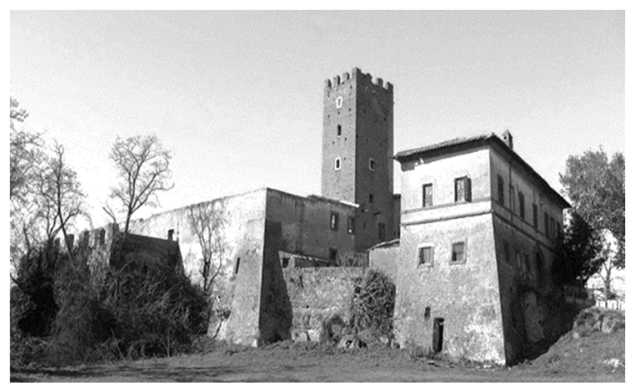

Fig. 1. Fotografia del Casale-Torre della Cervelletta. Nel caso specifico è presente una torre al centro del corpo di fabbrica.

Il casale Torre della Cervelletta a Tor Cervara è un esempio dello sviluppo di casali fortificati a una torre all'interno del territorio romano. La fortificazione del casale risale al XII secolo, esso rappresenta tutte le caratteristiche che definiscono questa tipologia edilizia. Ad oggi risulta in uno stato di abbandono, i corpi di fabbrica che lo compongono sono mancanti di copertura e alcune sue parti risultano inagibili.

Un'altra caratteristica di queste architetture è che la struttura muraria più moderna, datata come detto precedentemente tra il XII e il XIV secolo, si fonda su architetture preesistenti romane. (Esposito, 2005) Capita spesso, infatti, che i casali sorgano su preesistenze, in alcuni casi adottandone le originali funzioni, come per le cisterne. In altri casi i ruderi diventano fondazioni per le nuove muratore, mentre i vecchi locali ipogei trasformati in magazzini. Come accadeva spesso 
a Roma, i resti romani diventano cave a cielo aperto per il recupero di materiali da utilizzare nella costruzione dei casali, ed è comune ritrovare, incastonati nelle nuove murature, pezzi di travertino, marmo e decorazioni utilizzati per inframezzare i ricorsi di mattoni o per architravi e cornici di porte e finestre.

\section{Il caso studio: il Castellaccio di Monteroni}

Lo studio di questa tipologia architettonica è stato sviluppato attraverso un'analisi prima storica e poi geometrica del Castellaccio di Monteroni a Ladispoli. La scelta è ricaduta su questa specifica architettura, sia per il suo stato di conservazione sia per le sue peculiarità architettoniche che lo rendono un elemento unico all'interno della tipologia del casale (Fig. 2).

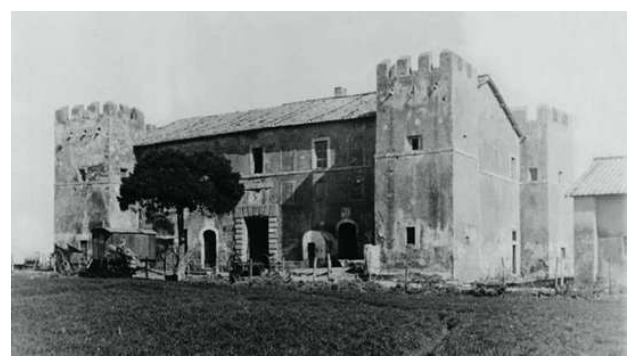

Fig. 2. Fotografia storica del Castellaccio di Monteroni. L'immagine mostra il casale prima dei lavori di restauro. Immagine tratta da dimorestorichelazio.it.

Il Castellaccio è un esempio di fortificazione medioevale del XIV secolo. Le quattro torri angolari, elemento caratteristico dell'architettura, sono postume rispetto all'assetto originario del casale. Il casale sorge sul tracciato dell'antica Via Aurelia, oggi via dell'Acquedotto della Statua, all'altezza del $35^{\circ} \mathrm{km}$ da Roma, nel centro della zona etrusco-romana dei Monteroni. Si posiziona in una zona di pregio storico architettonico tra l'Oasi di Paolo, Castello Odescalchi di Palo Spaziale e la necropoli di Monterone-. Il casale prende il proprio nome dalla necropoli adiacente. La necropoli con gli alti tumuli ne definisce il paesaggio circostante. Il nome Castellaccio invece deriva dallo stato di conservazione in cui versava dopo la II Guerra Mondiale. In quell'occasione venne utilizzato come rifugio per le famiglie sfollate di Ladispoli e come deposito, dopo la guerra divenne proprietà dell'Ente Maremma. Appartenuto in origine allo Stato Pontificio, nel XVI secolo diventa proprietà dei Marchesi di Riano e nel XVIII secolo di Livio III Odescalchi duca di Bracciano. Il casale nel corso della sua storia fu più volte ristrutturato, in seguito ai cambi di proprietà e di destinazione d'uso; l'ultimo restauro risale al 2000 in occasione del Giubileo. Il progetto fu curato dalla Provincia di Roma e dalla Soprintendenza ai Beni Culturali. Il casale svolse per secoli la funzione di stazione di sosta, rappresentando un punto sicuro di riferimento per pellegrini, corrieri e viaggiatori che percorrevano la via Aurelia. L'ultima funzione, prima della dismissione della struttura, fu quella di osteria e stazione di posta. Il Castellaccio di Monteroni è uno dei pochi esempi rimasti di casale-fortezza. Lo stato attuale lo rende una testimonianza inestimabile rispetto alle architetture dei casali della campagna romana (Fig. 3). Il corpo di fabbrica ha un ingombro planimetrico di $25 \times 31 \mathrm{~m}$ e un'altezza di 13 $\mathrm{m}$. Le fondazioni sono su resti romani probabilmente appartenenti a un mutatio $^{1}$ a guardia dell' antico tracciato dell' Aurelia. Infatti, i saggi svolti da Maria Cristina Rocco nel 2016, hanno portato alla luce una parte dell'antico tracciato della Via Aurelia alla quota archeologica.

La differenza principale rispetto ai casali tradizionali della campagna romana è la struttura architettonica delle torri (Ait, 2003). Solitamente, come spiegato nel paragrafo precedente, i casali presentavano una o due torri, il Castellaccio presenta 4 torri angolari, seguendo uno schema planimetrico di una vera e propria fortezza; da qui la definizione casale-fortezza. Le torri sono merlate, come da tipologia, ma presentano, rispetto agli altri casali, uno sviluppo in alzato minore. Inoltre, il casale risulta composto da due corpi di fabbrica principali, una struttura annessa e le quattro torri, ma originariamente era presente anche un fienile che ad oggi è andato perduto. Il manufatto presenta quindi in pianta un assetto più semplice rispetto ai tradizionali casali, per via della poca aggregazione di volumi, ma più complesso per quanto riguarda la geometria delle torri. L'aspetto materico dell'architettura mette in evidenza non solo le diverse funzioni del casale, ma anche la temporalità con cui sono sta- 
te costruite le varie parti. Il corpo principale è composto da una struttura in muratura con solai in legno e rifinita esternamente dall'intonaco. Le quattro torri angolari invece sono costruire con pietre Lysch della Tolfa, una pietra che è facile reperire nell'Appennino Centrale. La loro struttura, è assestante rispetto a quella del corpo di fabbrica principale e poggia su di esso.

Internamente l'architettura è caratterizzata da un alternarsi di solai a volte a botte e solai in legno al piano terra. Si sviluppa su tre piani con un mezzanino che occupa soltanto una porzione dell'edificio (Fig. 4).

\section{Lo studio geometrico e materico}

Il Castellaccio si presenta come un edificio a pianta rettangolare, con un corpo scale centrale e un altro elemento rettangolare di servizio posto nella parte posteriore. Le torri sono basate su un poligono a quattro lati irregolare, e si innestano sulla muratura originaria del casale, andando a caratterizzare gli angoli dell'architettura creando un manufatto ibrido da casale e castello. Le torri a differenza del tradizionale casale non superano in altezza il corpo di fabbrica ma sono leggermente più basse rispetto al colmo della coper- tura, mettendo in evidenza che lo scopo principale di esse non era di avvistamento ma di difesa. Questa particolarità è probabilmente attribuibile ai residenti di rango più alto nel corso dei secoli rispetto ai tradizionali casali che erano di proprietà dei mercanti.

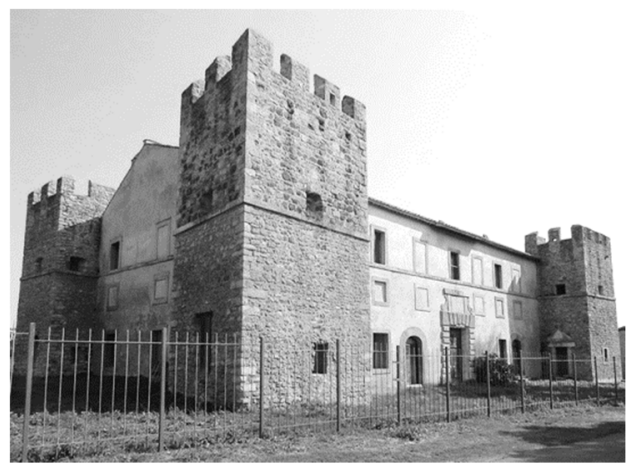

Fig. 3. Fotografia del 2018 del Castellaccio di Monteroni. Vista dell'ingresso principale.

Il rilievo è stato un elemento di conoscenza fondamentale per poter comprendere l'apparato architettonico del Castellaccio. Il rilievo è uno strumento indagatore, di analisi e conoscenza del manufatto architettonico. Il suo scopo è quello di restituire l'immagine dell'opera, rappresentata in

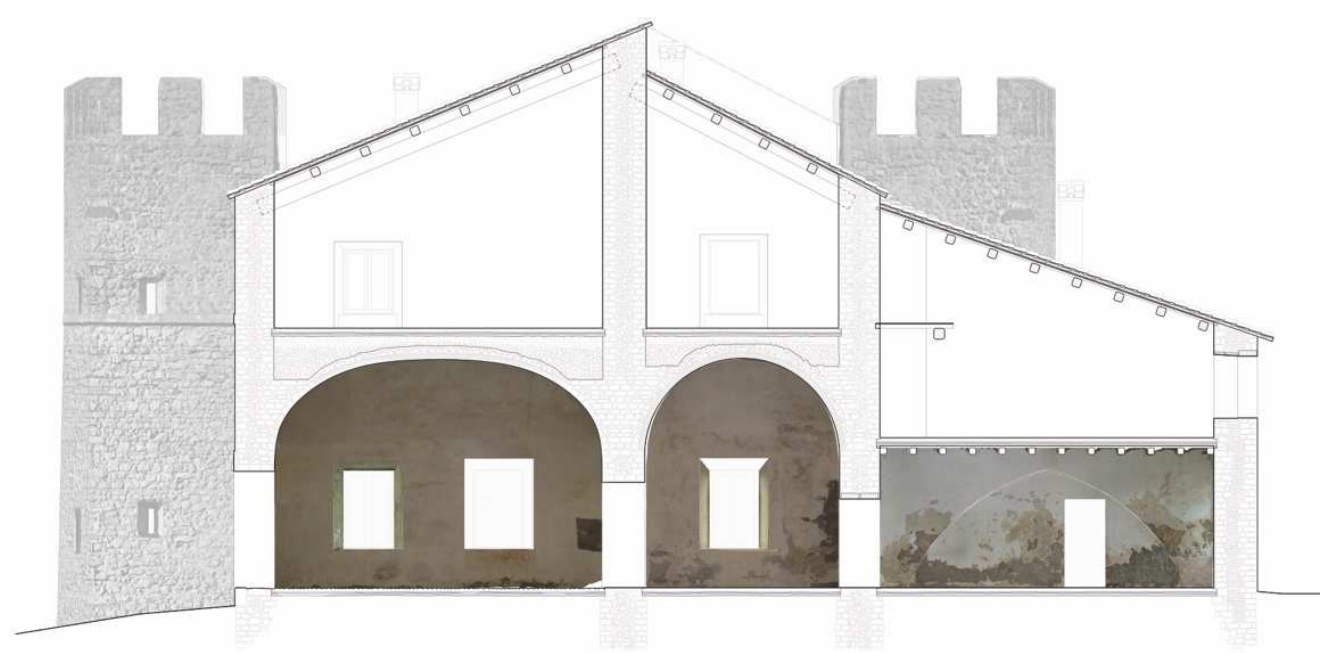

Fig. 4. Sezione Trasversale dell'edificio. Si possono notare le differenti quote degli impalcati e le diverse tecnologie utilizzate. L'elaborato è stato realizzando integrando elementi ottenuti tramite rilievo diretto e rilievo strumentale. 
modi differenti a seconda dello scopo ultimo. La rappresentazione del manufatto e il relativo processo di rilievo sono un processo soggettivo. Il rilevatore e rappresentatore sviluppa un processo di discretizzazione per scegliere gli elementi consoni da rilevare. Il processo di discretizzazione si ritrova poi nella rappresentazione del rilievo attuato, dove a seconda del fine ultimo vengono messe in luce le diverse parti dell'architettura. Il rilevamento architettonico risulta quindi non solo uno strumento per la misurazione di un edificio o di un contesto urbano, ma un'operazione di conoscenza molto complessa che fa interagire diverse discipline e che utilizza strumenti e mezzi adeguati e molteplici.

Il rilievo dell'edificio è stato svolto utilizzando la metodologia del rilievo integrato, tecniche tradizionali del rilievo diretto con le più moderne tecnologie del rilievo strumentale. ${ }^{2}$ Lo studio geometrico (Fig. 5) risulta di grande importanza per poter comprendere in che modo il casale si sia sviluppato e trasformato nella sua configurazione non solo planimetrica ma anche in alzato nel corso dei secoli. Il rilievo si è svolto in tre fasi, una prima fase basata sulla conoscenza storico culturale dell'edificio, analizzando per analogia altri casali sul territorio e le conseguenti differenze tipologiche. Una seconda fase ha compreso la conoscenza geometrica dell'edificio, mentre una terza fase si è basata sulla consapevolezza materica dell'opera e delle tecniche costruttive utilizzate nella realizzazione.

La prima fase si è attuata attraverso il rilievo diretto e strumentale utilizzando la tecnica della fotomodellazione. Con il rilievo geometrico, attraverso la tecnica della trilaterazione e della misurazione dei singoli elementi si è riprodotto l'assetto planimetrico e dei singoli dettagli dell'edificio. Un assetto complesso, sia per i cambi di quota che per la difficile accessibilità di alcuni luoghi. In parallelo sono state attuate delle campagne di rilevamento fotografico in modo da poter poi utilizzare la tecnica della fotomodellazione per compensare le mancanze e di conseguenza implementare il rilievo diretto. Attraverso le tecniche SfM (Structure from Motion), che permettono di ricostruire le geometrie 3D con l'accoppiamento di due o più foto in cui vengono riconosciuti dei punti omologhi, è stato possibile ricreare un modello tridimensionale dell'oggetto caso studio (Fig. 6) (Luca, 2011). I punti omologhi sono elementi facilmente riconoscibili in coppie di fotogrammi.

Questo modello ha permesso di studiare più nello specifico il rapporto tra pianta e alzato e di semplificare la rappresentazione della matericità dell'architettura. Il degrado della parte intonaca esterna e la superficie in pietra presente sulle torri, ha permesso una ricostruzione puntuale e meticolosa dei prospetti dell'apparato architettonico. All'interno dell'edificio le diverse tipologie di luce, le diverse finiture chiare delle pareti e in alcuni casi il basso degrado dell' architettura non ha permesso una ricostruzione completa di ogni singolo ambiente. La campagna fotografica, per la digitalizzazione del prospetto esterno ha richiesto 350 fotografie. L'alto numero di fotogrammi è dovuto sia alla grandezza del manufatto, sia ai gradi di dettaglio sulle diverse parti dell'edificio, dove la comprensione tridimensionale dell'opera richiedeva uno studio più accurato. Sono stati realizzati per questo, cinque modelli digitali: uno generale e quattro per le torri d'angolo. Le nuvole di punti dense, derivanti dall'accoppiamento di punti omologhi, sono state successivamente unite in un unico modello con un alto grado di dettaglio. La ripresa del corpo principale è stata realizzata sfruttando la tecnica degli assi paralleli, che consiste in fotogrammi paralleli all'edificio da rilevare con una sovrapposizione verticale del $60 \%$ e orizzontale del $30 \%$. La sovrapposizione ha permesso di avere una quantità sufficiente di punti in comune per ricreare digitalmente l'architettura. L'utilizzo di più nuvole di punti permette di ridurre notevolmente i tempi di calcolo per realizzare i modelli digitali. La fotomodellazione è stata fondamentale per realizzare degli ortofotopiani volti al rilievo materico e all'individuazione del degrado dell'edificio (Fig. 7).

L'uso delle ortofoto nel campo del progetto di restauro è di grande importanza, tuttavia bisogna anche ricordare che le decisioni sulla metodologia di rappresentazione influenzano fortemente la qualità non solo dei disegni, ma anche la loro 
facile comunicazione al cliente, alla ditta, alle soprintendenze. L'ortofotopiano può essere d'ausilio e integrato al rilievo architettonico. Ldiscretizzazioni necessariamente eseguite dal progettista, ad esempio, per la visualizzazione dell'apparato murario superficiale sui prospetti, introduce una semplificazione rappresentativa. Le ortofoto non sono soggette a questo processo: l'ortofoto restituisce esattamente tutto quello che è stato oggetto della campagna fotografica e alla base della creazione del modello tridimensionale. Il software non esegue una scelta di informazioni da mostrare, tuttavia, sempre su richiesta dell'uomo, può abbassare la risoluzione dell'immagine con conseguente perdita di dettaglio e anche di alcuni dati.

Il processo di semplificazione, pertanto, sarà di perdita della qualità dei dati e non della quantità. È pertanto opportuno mantenere elevata la qualità delle ortofoto e attuare su di esse il processo di selezione critica sui disegni. Dagli ortofotopiani è poi possibile successivamente realizzare degli elaborati che permettono di esemplificare i materiali che vanno a comporre l'architettura e il relativo degrado (Fig. 8).

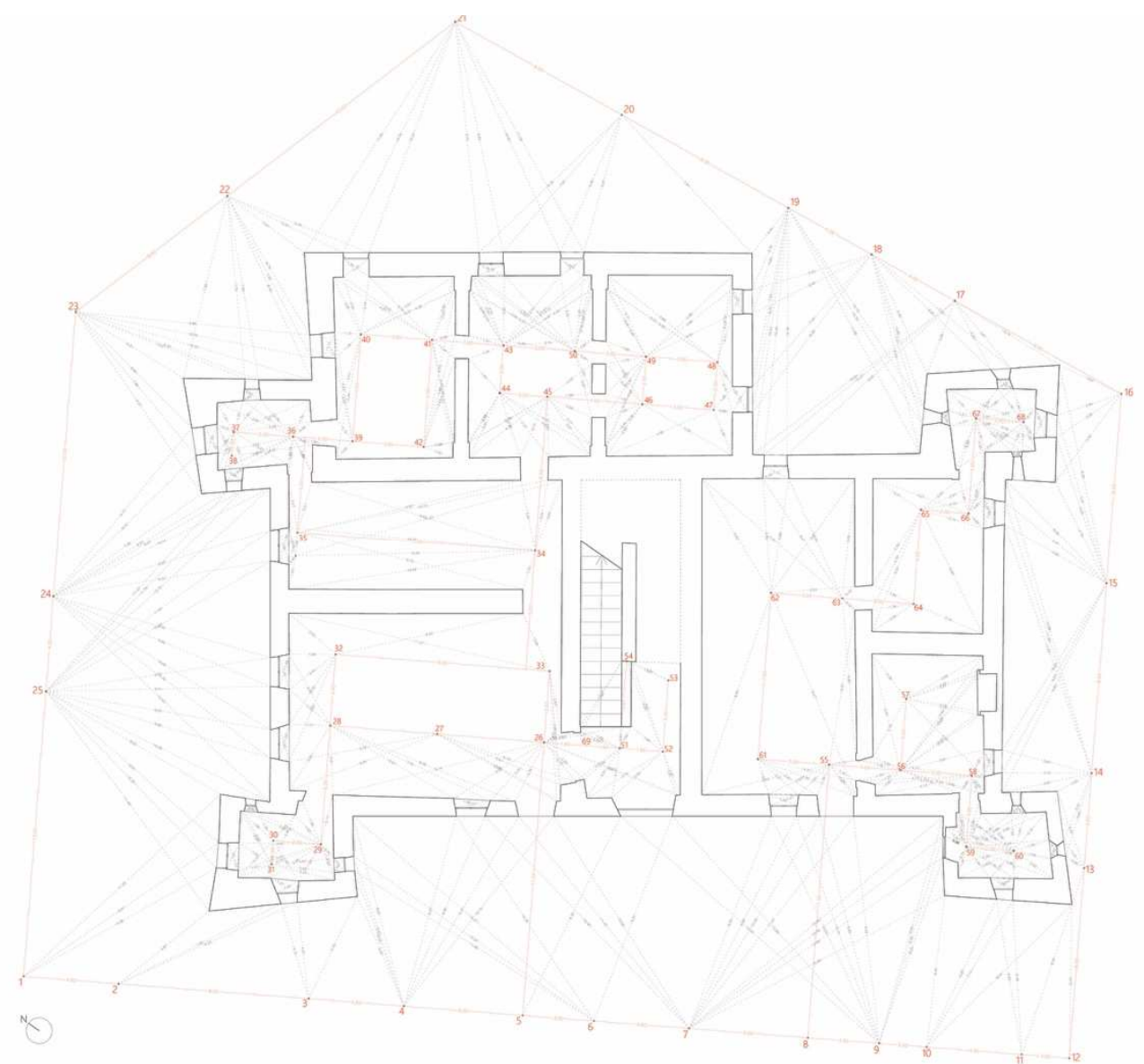

Fig. 5. Pianta del piano terra, rilievo geometrico dell'edificio. In rosso la fondamentale attraverso la quale è stato attuato il rilievo. 


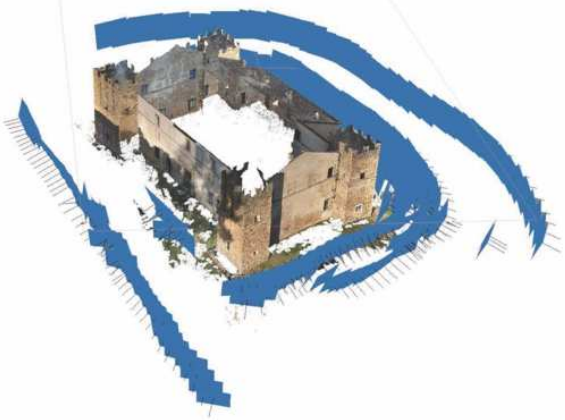

Fig. 6. Modello tridimensionale del Castellaccio. In evidenza le camere utilizzate per poter ricreare la geometria esterna del manufatto.

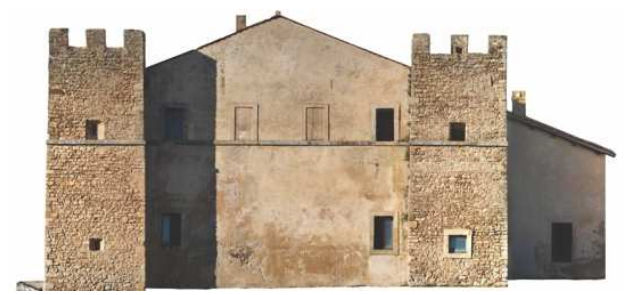

Fig. 7. Ortofotopiano realizzato da fotomodellazione. Prospetto sud-est.
Il processo di conoscenza, attuato tramite la metodologia del rilievo integrato, ha permesso non solo di redigere elaborati tematici volti a una conoscenza architettonica dell'edificio ma soprattutto a redigere una solida base per un progetto di restauro dell'edificio.

Parallelamente alla ricerca materica e del degrado dell'edificio è stato svolto uno studio sulle tecniche costruttive, redigendo degli elaborati volti alla conoscenza tecnologica utilizzata nella progettazione del Castellaccio.

\section{Conclusioni}

Il caso studio del Castellaccio di Monteroni è un elemento architettonico di grande interesse per la comprensione dei manufatti della campagna romana. L'importanza del Castellaccio non deriva solamente dalla sua peculiarità architettonica, che per assetto planimetrico e struttura difensiva lo differenzia notevolmente dai tradizionali resti dei casali della campagna romana, ma anche e soprattutto perché è uno dei pochi casi rimasti che narra la storia dell'evoluzione stilistica di queste architetture.

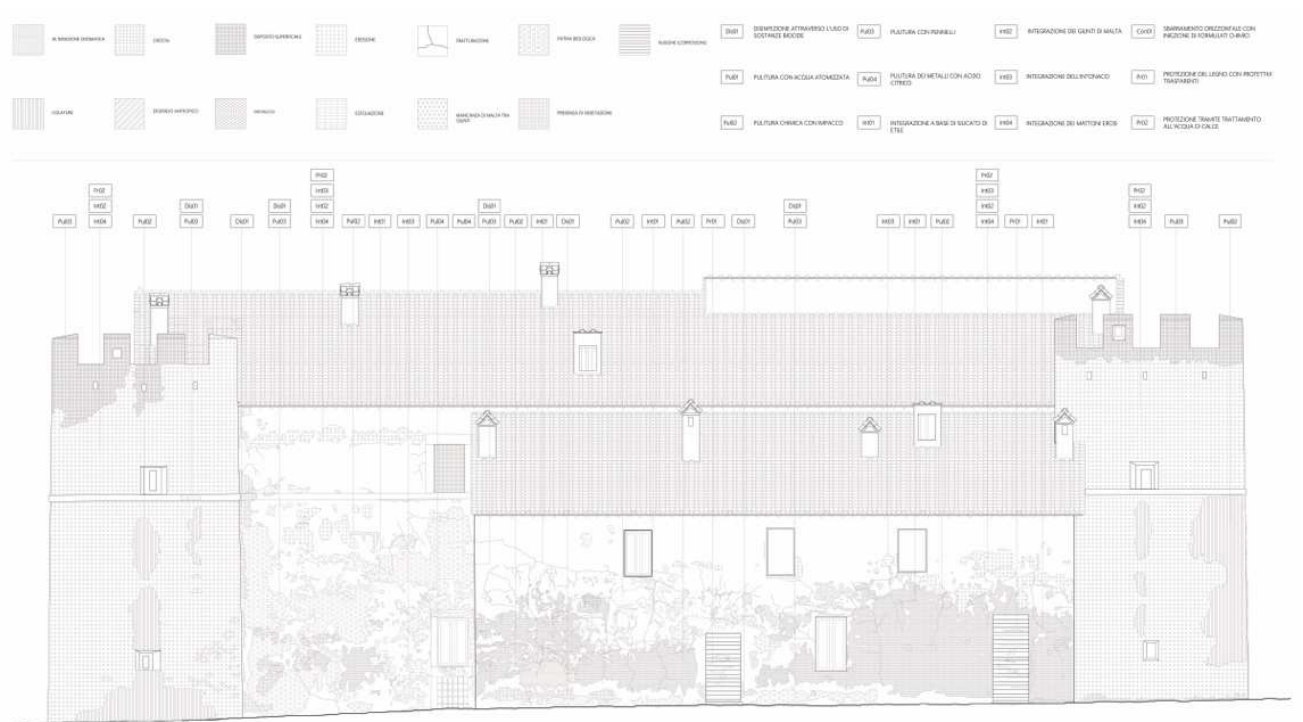

Fig. 8. Prospetto sud-est dell'edificio. Elaborato che esemplifica i degradi presenti esternamente con tipologia di tecnica da attuare per il restauro dell'edificio. Il degrado è stato mappato tramite la sovrapposizione con gli ortofotopiani realizzati. 
Le differenze stilistiche che caratterizzano l'architettura del Castellaccio di Monteroni come una vera e propria fortezza, lo rendono, un elemento di interesse soprattutto per la presenza delle quattro torri angolari con funzione difensiva. Questa tipologia di casale-fortezza è in netto contrasto con la presenza di una torre per manufatto, caratteristica principale della campagna romana. Lo studio del Castellaccio di Monteroni si inserisce all'interno di una ricerca più ampia volta ad analizzare le architetture rurali della campagna romana. Questa ricerca mira a comprendere non solo le metodologie di rilievo più idonee per il rilevamento di un' architettura rurale ma anche a comprenderne le evoluzioni stilistiche avvenute nel corso dei secoli. Rimarcare l'importanza di queste architetture storiche della campagna romana, ha lo scopo di portare l'attenzione su questi edifici che nel corso dei secoli hanno subito una politica di abbandono
(Calisi, Zappa, 2019). Lo stesso Castellaccio ha subito un restauro recente, ma il disinteresse delle amministrazioni, non ha permesso un suo riutilizzo facendolo ricadere in uno stato di degrado. Dove architetture più simboliche vengono salvaguardate, le archeologie rurali vengono largamente lasciate in uno stato di degrado, non tutelate con la stessa cura e sottovalutandone il valore storico culturale.

\section{Note}

${ }^{1}$ Muatatio. Dopo la Caupona e Taberna Un terzo sistema di "stazioni di servizio" per veicoli e animali: le mutationes (stazioni di cambio) si trovavano a intervalli di 12-18 miglia.

${ }^{2}$ Il rilievo è stato svolto grazie al prezioso aiuto di: Alina Kruk, Roberto Mariani, Michela Masotina, Carmen Melchionna, Sara Pasquale, Chiara Trebb.

\section{Bibliography}

Ait, I. (2003). "Roma: una città in crescita tra strutture feudali e dinamiche di mercati, in Le città del Mediterraneo all'apogeo dello sviluppo medievale: aspetti economici e sociali”, in Atti del diciottesimo Convegno internazionale di studi, Pistoia, 18-21 maggio 2001, Pistoia.

Bagnarini, N. (2013). "Tra la costa, la città ed il contado: castelli, domus e casali fortificati nel Lazio Templare. Studio storico ed analisi architettonica", in Ferreira Fernandes, I.C. ed., Castelos das Ordens Militares, vol. II, pp. 99-118.

Calisi, D.; Zappa, A. (2019). "The farmhouses of the roman countryside: census and catalog. The case of the estate of Farnesiana", The International Archives of the Photogrammetry, Remote Sensing and Spatial Information Sciences, XLII-2/W15, pp. 223-230.

Calzolari, V. (1999). Storia e natura come sistema: un progetto per il territorio libero dell'area romana, Argos, Roma.

Carocci, S.; Vendittelli, M. (2004). L'origine della Campagna Romana: Casali, castelli e villaggi nel 12. e 13. Seco$l o$, Società romana di storia patria, Roma.

Cosenza, F. (2017). "Il sistema dei Casali della Campagna romana: problemi e metodologie d'indagine", in III Ciclo di Studi Medievali. Atti del Convegno 8-10 settembre 2017, Firenze, pp. 189-203.

Coste, J. (1969). I casali della campagna romana all'inizio del Seicento, in Archivio R. Società Romana di Storia Patria, Roma, p. 92.

Esposito, D. (2005). Architettura e costruzione dei casali della Campagna Romana tra XII e XIV secolo, Arbor Sapientiae, Roma.

Luca, L. De. (2011). La fotomodellazione architettonica. Rilievo, modellazione, rappresentazione di edifici a partire da fotografie, Flaccovio Dario Ed.

Rossi, G.M. De. (1969). Torri e Castelli medievali della Campagna romana, De Luca per Sigma Tau, Roma. 